\title{
Defining Priority Areas for Critical Care Simulation: A Modified Delphi Consensus Project
}

Ilana Harwayne-Gidansky ${ }^{1}$, Adrian Zurca ${ }^{2}$, Tensing Maa ${ }^{3}$, Utpal S. Bhalala ${ }^{4}$, Deepa Malaiyandi ${ }^{5}$, Pooja Nawathe ${ }^{6}$, Aarti Sarwal ${ }^{7}$, Muhammad Waseem ${ }^{8}$, Michael Kenes ${ }^{9}$, Megan Vennero ${ }^{10}$, Lillian Emlet ${ }^{11}$

1. Pediatrics, Stony Brook Children's Hospital, Stony Brook, USA 2. Pediatric Critical Care Medicine, Penn State Health Children's Hospital, Hershey, USA 3. Pediatric Critical Care Medicine, Nationwide Children's Hospital, Ohio State University College of Medicine, Columbus, USA 4. Pediatric Critical Care Medicine, Children's Hospital of San Antonio, San Antonio, USA 5. Neuro-critical Care, University of Toledo, Toledo, USA 6. Pediatric Critical Care Medicine, CedarsSinai Medical Center, Los Angeles, USA 7. Neurology, Wake Forest School of Medicine, Winston-Salem, USA 8. Pediatric Emergency Medicine, Lincoln Medical \& Mental Health Center, New York, USA 9. Pharmacy, University of Michigan, Ann Arbor, USA 10. Emergency Medicine, Veterans Affairs Pittsburgh Healthcare System, Pittsburgh, USA 11. Emergency Medicine, University of Pittsburgh Medical Center, Pittsburgh, USA

Corresponding author: Ilana Harwayne-Gidansky, ilanahg@mac.com

\section{Abstract \\ Background}

Simulation is used in critical care for skill development, formative assessment, and interprofessional team performance. Healthcare educators need to balance the relatively high cost to deliver simulation education with the potential impact on healthcare quality. It is unclear how to prioritize simulation in critical care education, especially considering interprofessional needs across adult and pediatric populations. The objective of this study was to prioritize topics for critical care educators developing simulation-based educational interventions.

\section{Methodology}

A modified Delphi process was used to identify and prioritize critical care topics taught using simulation. We disseminated a multi-institutional survey to understand critical care simulation topics using a three-round modified Delphi technique. An expert panel was recruited based on their expertise with simulation-based education through the Society for Simulation in Healthcare and the Society of Critical Care Medicine lists. Critical care topics originated using content derived from multiple critical care board examination contents. Additional content for a critical care simulation-based curriculum was generated.

\section{Results}

Consensus and prioritization were achieved in three rounds, with 52 simulation experts participating. The first Delphi round surveyed priority topics in critical care content and generated additional topics for inclusion in round two. The second Delphi round added the content with the highest-ranked items from round one to generate a set of simulation-based topic priorities. The third Delphi round asked participants to

Review began 06/08/2021 Review ended 06/12/2021 Published 06/22/2021

\section{() Copyright 2021}

Harwayne-Gidansky et al. This is an open access article distributed under the terms of the Creative Commons Attribution License CC-BY 4.0., which permits unrestricted use, distribution, and reproduction in any medium, provided the original author and source are credited. determine the importance of each priority item taught via simulation compared to other modalities for clinical education. This round yielded 106 topics over four domains categorized into (1) Diagnosis and Management of Clinical Problems, (2) Procedural Skills, (3) Teamwork and Communication Skills, and (4) General Knowledge and Knowledge of Technical Adjuncts.

\section{Conclusions}

The modified Delphi survey revealed a prioritized, consensus-based list of topics and domains for critical care educators to focus on when creating a simulation-based critical care curriculum. Future work will focus on developing specific simulation-based critical care curricula.

Categories: Medical Education, Medical Simulation, Other

Keywords: simulation, medical education, training, pulmonary critical care, critical care, pediatric emergencies and critical care, interprofessional education and collaboration

\section{Introduction}

Simulation-based medical education (SBME) is widely accepted as an effective method to teach interprofessional and interdisciplinary team communication skills, technical skills, high-stakes rare events, and integrative case-based demonstration of higher-level skills [1]. Healthcare professionals across the continuum need mastery of these skills and core competencies from undergraduate medical education (UME) to postgraduate training in multiple healthcare disciplines and specialties. In critical care, simulation has historically focused primarily on resuscitation and procedural skills. 
Evidence supports using SBME to teach numerous domains of critical care, spanning across multiple medical specialties and healthcare professions [2-4]. Educators and healthcare systems must balance the financial and personnel costs of SBME with the effectiveness of reaching desired learning and healthcare outcomes [5]. Although SBME is resource-intensive, its integration in curricula has dramatically expanded its reach, demonstrating improvements to patient-driven outcomes [6-8]. These include just-in-time infant lumbar puncture, simulation-based mastery learning of central venous line (CVL) placement, and using virtual reality to teach patient assessments [9-13].

Despite the evidence of its importance, there is no current widespread standardized curriculum or guidance on critical care simulation content for educators. Although several disciplines have developed guidelines for teaching critical care topics, none have exclusively prioritized the role of a simulation-based curriculum for this level of intensive care training $[14,15]$. Therefore, it remains unclear how to prioritize topics best suited to be taught via simulation for critical care healthcare professionals. Thus, we aimed to assess the current status of SMBE in critical care and develop priority areas for the effective and efficient utilization of simulation for critical care education.

\section{Materials And Methods}

\section{Design and Delphi process}

A modified Delphi was used to develop consensus around SBME priorities and present a list of content to guide future simulation-based educational priorities such as generating a standard critical care simulation curriculum [14-16]. The Delphi method relies on expert opinion to arrive at these conclusions. A six-step approach is adopted when utilizing this process: (1) stating the problem, (2) developing an initial questionnaire, (3) selecting experts, (4) iteratively polling experts, (5) identifying potential consensus points, and (6) reporting results. This approach allows for an iterative understanding of the question with feedback given by experts between cycles [16-18] (Figure 1).

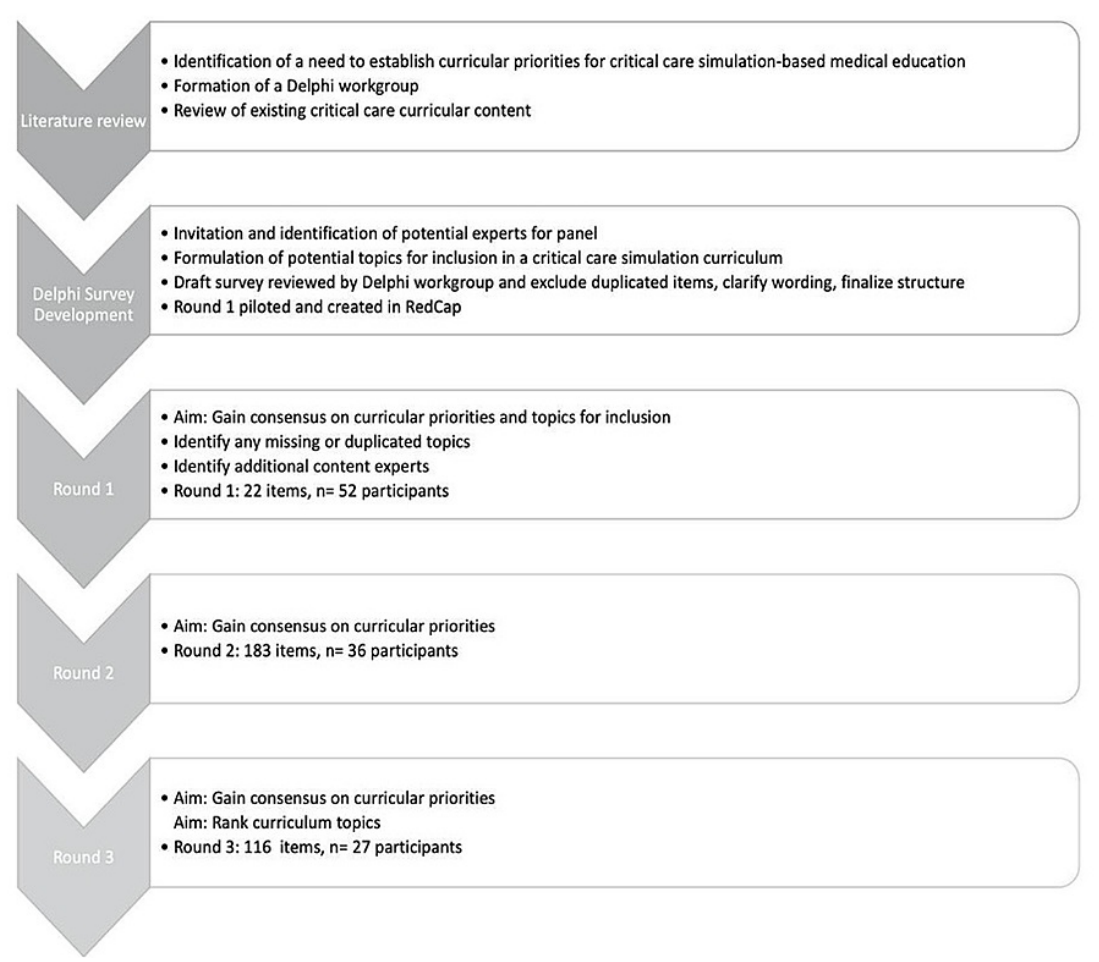

FIGURE 1: Methodological process for generating prioritized simulationbased critical care content.

\section{Identification of simulation education experts for Delphi panel}

The research team was composed of interprofessional members of the Society of Critical Care Medicine (SCCM) Research Section, Simulation Education Research Subcommittee. Simulation education experts were identified through the purposeful sampling of publicly available lists of simulation centers to identify experts in critical care simulation education for this panel. Representatives of accredited simulation centers available through the American Board of Anesthesiology and Society for Simulation in Healthcare were approached, along with members of the SCCM Education Research Committee. We defined "critical care simulation educator" as someone who uses any modality of technology-enhanced simulation to educate 
learners, utilizing evidence-based strategies directed at critical care practice objectives [19]. We established a panel of 52 experts from multiple professions consistent with the standard Delphi procedure. A panel of interprofessional content experts included physicians, nurses, educators, and pharmacists. The snowball method was used to sample participants, where each participant was asked in the initial survey to identify additional expert participants who then also received an invitation to participate in the study [20]. Experts were sent an email invitation explaining the study's purpose and a link to the survey for the first round of the Delphi. Participation was voluntary, and this study was approved by the University of Pittsburgh Institutional Review Board as a non-human subjects exempt survey research study. The participants remained anonymous to each other throughout the study.

Study data were collected and managed using REDCap electronic data capture tools co-hosted at the University of Pittsburgh and Stony Brook University. Surveys were distributed between December 2018 and June 2019. Responses were collected and de-identified for final data analysis. No limits on respondents were set a priori. Once panelists agreed to participate, a link to the first round of the survey was sent, along with detailed instructions on how to complete the Delphi surveys.

The writing group consisted of 12 members, a diverse interprofessional and interdisciplinary group of simulation educators and facilitators from neurocritical care (2), pediatric critical care (5), emergency medicine-critical care (1), pediatric emergency medicine (1), critical care nurse practitioners (1), and critical care pharmacist (1).

\section{Survey design and iterative feedback}

Initial first-round items were derived from blueprint content of critical care board examinations from the following specialties: the American Board of Internal Medicine, American Board of Surgery, American Board of Pediatrics, American Board of Emergency Medicine, and American Board of Anesthesiology. Descriptions of training program requirements in critical care medicine were reviewed to determine a list of topics for ranking in the survey. As all study team members were critical care simulation educators, a pilot test of the survey for readability, feasibility, and face validity was performed, and changes were made as needed. Experts were asked to prioritize each topic on a three-point Likert scale (least important, somewhat important, most important). Free text options to describe the current simulation curriculum and its associated objectives were included. At the end of the first round, unique free-text items were analyzed and included in the second round as potential additions to curricular content.

Consensus was defined a priori as $>80 \%$ agreement with "somewhat" or "most" important selected. Topics with a consensus of $<80 \%$ were not included in the priority list for round two as they were thought to be unlikely to reach a consensus. Round two items were aggregated from round one, and potential topics for inclusion in a critical care simulation content outline were included in the final round of content generation. At the end of the second round, we selected the items from the list of consensus curriculum that achieved this threshold [16-18,21]. Following this round, we formulated potential consensus items for inclusion into the third round. This method was used, in part, to assure a more comprehensive capture of content.

For round three, items were again aggregated and then prioritized into three domains of content that were most important, somewhat important, or least important to include as SBME content. For each ranking, we asked participants to rate each topic using a three-point Likert scale (with options labeled 1 = best taught via simulation to 3 = should not be taught via simulation).

To obtain a prioritized list of critical care content, we used the methods proposed by Altschuld and Thomas, where content is ranked and scored according to the frequency of occurrence for prioritization scoring [22]. According to this method, minimum priority was defined as a prioritization score of $>50$, with strong prioritization $>60$. Those topics not included in the priority list scored $<50$ were classified as low priority and not included.

\section{Outcome measures}

The primary outcomes of interest were ranked in order of importance of areas of critical care content. We defined "simulation education" as any modality of technology-enhanced (computer, manikin, standardized patient, excluding animal, tissue, or cadaveric models) simulation, which is an educational tool with which the learner interacts to mimic an aspect of clinical care for the purpose of teaching or assessment in the delivery of critical care to adult or pediatric patients [19]. The primary outcome of the third round Delphi was to generate a list of prioritized content deemed important by this multi-professional group of critical care simulation experts.

Consensus was reached after round three, from which we developed a critical care simulation content outline. All recommended topics were mapped into four main learning domains: General Knowledge, Diagnosis and Management, Procedural Skills, and Teamwork and Communication; consensus was reported among all simulation specialists (Figure 2). 


\section{Cureus}

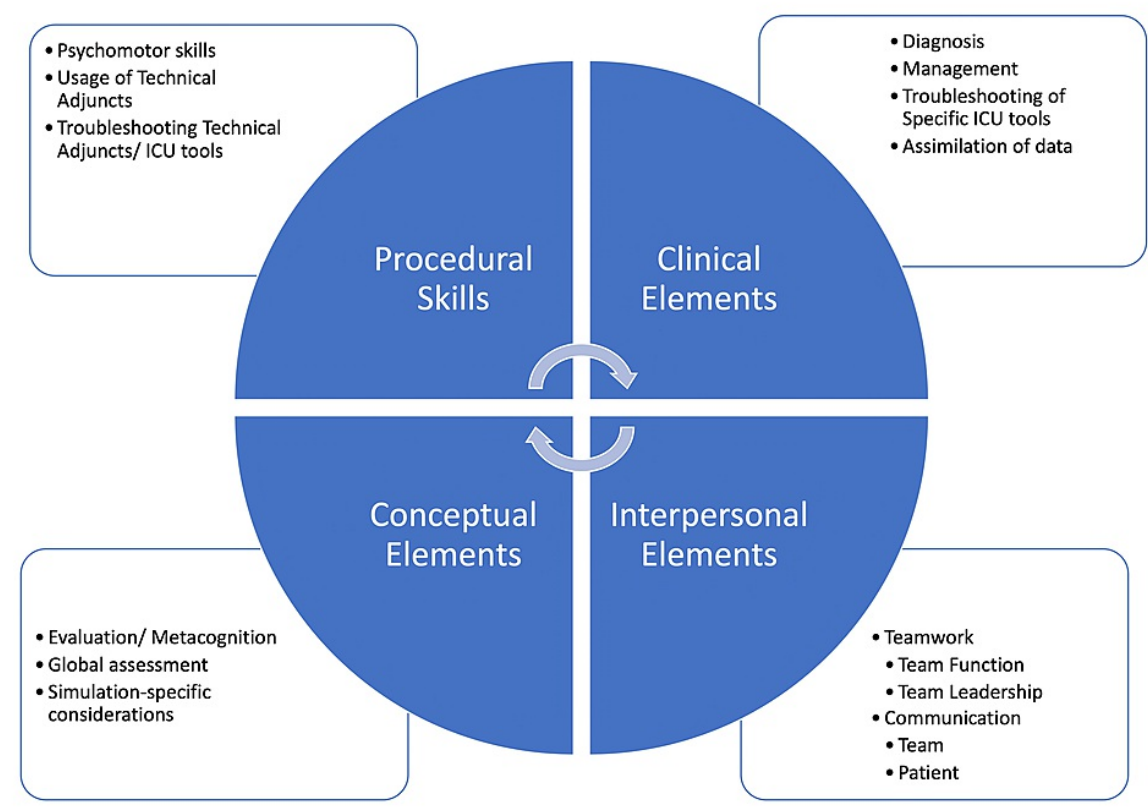

\section{FIGURE 2: Priority domains for critical care simulation.}

ICU: intensive care unit

\section{Results}

A total of 52 experts participated in the initial survey, 27 of whom completed all three rounds (52\%). The majority of participants worked in critical care. There were $34(65 \%)$ respondents who were physicians, followed by nurse practitioners ( $n=6,12 \%)$, nurses $(n=5,10 \%)$, pharmacists $(n=4,8 \%)$, respiratory therapists $(n=2,4 \%)$, and other $(n=1,2 \%)$. Experts most commonly represented pediatrics $(n=14,27 \%)$ and emergency medicine $(n=9,17 \%)$. These experts reported working in 16 states across all geographical

regions of the United States. Additional demographic data of the simulation experts are presented in Table 1. 


\section{Cureus}

\begin{tabular}{|c|c|c|c|}
\hline & & $\begin{array}{l}N= \\
52\end{array}$ & (\%) \\
\hline \multirow{3}{*}{ Please list your primary patient population } & $\begin{array}{l}\text { Pediatric patients (including } \\
\text { neonatology) }\end{array}$ & 20 & $(41.6 \%)$ \\
\hline & Adult patients & 25 & $(52.0 \%)$ \\
\hline & Other (please specify) & 3 & $(6.3 \%)$ \\
\hline \multirow{6}{*}{ Profession } & Physician & 34 & $(65.4 \%)$ \\
\hline & Nurse & 5 & $(9.6 \%)$ \\
\hline & Respiratory therapist & 2 & $(3.8 \%)$ \\
\hline & Pharmacist & 4 & $(7.7 \%)$ \\
\hline & Nurse practitioner & 6 & $(11.5 \%)$ \\
\hline & Other (please specify) & 1 & $(1.9 \%)$ \\
\hline \multirow{9}{*}{ In which clinical area do you primarily work? } & Neuro-intensive care & 3 & $(5.8 \%)$ \\
\hline & Emergency medicine & 9 & $(17.3 \%)$ \\
\hline & Cardiovascular intensive care & 2 & $(3.9 \%)$ \\
\hline & Trauma/surgical intensive care & 5 & $(9.6 \%)$ \\
\hline & Anesthesiology & 3 & $(5.8 \%)$ \\
\hline & Pediatrics & 14 & $(26.9 \%)$ \\
\hline & Other & 7 & $(13.5 \%)$ \\
\hline & Medical intensive care & 5 & $(9.6 \%)$ \\
\hline & Nonclinical & 4 & $(7.7 \%)$ \\
\hline \multirow{5}{*}{$\begin{array}{l}\text { How many years have you worked in your current profession (excluding } \\
\text { trainee time)? }\end{array}$} & $0-5$ & 4 & $(7.7 \%)$ \\
\hline & 6-10 & 20 & $(38.5 \%)$ \\
\hline & 11-15 & 6 & $(11.5 \%)$ \\
\hline & $16-20$ & 6 & $(11.5 \%)$ \\
\hline & $>20$ & 15 & $(28.8 \%)$ \\
\hline
\end{tabular}

TABLE 1: Demographic information.

The first round of the Delphi survey contained demographic information and 22 items focused on simulation, including free-text boxes for participants to add additional critical care curriculum content not previously captured. The second survey included 183 questions and with a completion rate of 69\% (36/52). The third and final survey generated a list of 116 elements of ranked content and was returned with a completion rate of $75 \%(27 / 36)$.

After the Delphi process, experts identified 106 items to be recommended for critical care content over four domains. Topics with a score of $>60$ were strongly prioritized in the four domains of General Knowledge and Knowledge of Technical Adjuncts, Diagnosis and Management, Procedural Skills, and Teamwork and Communication Skills, and are listed in Tables 2-5. 


\section{Cureus}

\begin{tabular}{|c|c|c|c|}
\hline Topic & Category theme & Score & $\begin{array}{l}\text { Overall rank } \\
\text { order }\end{array}$ \\
\hline Extracorporeal life support & $\begin{array}{l}\text { Procedural } \\
\text { Skills }\end{array}$ & 67 & 13 \\
\hline Intra-aortic balloon pump & $\begin{array}{l}\text { Procedural } \\
\text { Skills }\end{array}$ & 67 & 13 \\
\hline Ventricular assist devices & $\begin{array}{l}\text { Procedural } \\
\text { Skills }\end{array}$ & 67 & 13 \\
\hline Use of invasive neurologic monitoring (e.g., external ventricular drain and bolts) & $\begin{array}{l}\text { Procedural } \\
\text { Skills }\end{array}$ & 64 & 16 \\
\hline Continuous renal replacement therapy usage and troubleshooting & $\begin{array}{l}\text { Procedural } \\
\text { Skills }\end{array}$ & 62 & 18 \\
\hline Limitations of the simulation (e.g., proper pre-simulation preparation) & $\begin{array}{l}\text { Conceptual } \\
\text { Element }\end{array}$ & 62 & 18 \\
\hline Surgical technical adjuncts & $\begin{array}{l}\text { Procedural } \\
\text { Skills }\end{array}$ & 62 & 18 \\
\hline $\begin{array}{l}\text { Understanding how to use and troubleshoot respiratory technological adjuncts (e.g., } \\
\text { mechanical ventilation) }\end{array}$ & $\begin{array}{l}\text { Procedural } \\
\text { Skills }\end{array}$ & 62 & 18 \\
\hline Utilization of hemodynamic monitoring data in patient management & $\begin{array}{l}\text { Clinical } \\
\text { Elements }\end{array}$ & 62 & 18 \\
\hline Checking medications & $\begin{array}{l}\text { Clinical } \\
\text { Elements }\end{array}$ & 61 & 19 \\
\hline
\end{tabular}

TABLE 2: Strongly prioritized topics: General Knowledge and Knowledge of Technical Adjuncts Domain. 


\section{Cureus}

Topic

Cardiovascular/hemodynamic emergencies: management of patients in cardiac arrest

Diagnosis and management (e.g., clinical reasoning) during a code

Management of acute trauma resuscitation (e.g., blunt, penetrating)

Management of anaphylaxis

Management of bleeding emergencies (e.g., hemorrhage)

Diagnosis and management of shock states

Management of neurologic emergencies (e.g., intracerebral hemorrhage, status epilepticus, head trauma, spinal cord trauma)

Diagnosis of hyperthermic emergencies (i.e., malignant hyperthermia/neuroleptic malignant syndrome)

Cardiovascular diagnosis and management (e.g., arrhythmia, acute coronary syndrome, heart failure, pulmonary hypertension)

Cardiovascular/hemodynamic emergencies: management of patients in low cardiac output state (not cardiac arrest)

Management of infectious disease emergencies (e.g., septic shock)

Medical management of pericardial tamponade

Pulmonary emergencies (e.g., hemorrhage, edema)

Diagnosis and management of acute respiratory failure

Approach and management of bioterrorism

Gastrointestinal emergencies (e.g., gastrointestinal hemorrhage, esophageal perforation)

\begin{tabular}{|c|c|c|}
\hline $\begin{array}{l}\text { Category } \\
\text { theme }\end{array}$ & Score & $\begin{array}{l}\text { Overall } \\
\text { rank order }\end{array}$ \\
\hline $\begin{array}{l}\text { Clinical } \\
\text { Elements }\end{array}$ & 75 & 5 \\
\hline $\begin{array}{l}\text { Clinical } \\
\text { Elements }\end{array}$ & 73 & 7 \\
\hline $\begin{array}{l}\text { Clinical } \\
\text { Elements }\end{array}$ & 73 & 7 \\
\hline $\begin{array}{l}\text { Clinical } \\
\text { Elements }\end{array}$ & 73 & 7 \\
\hline $\begin{array}{l}\text { Clinical } \\
\text { Elements }\end{array}$ & 70 & 10 \\
\hline $\begin{array}{l}\text { Clinical } \\
\text { Elements }\end{array}$ & 68 & 12 \\
\hline $\begin{array}{l}\text { Clinical } \\
\text { Elements }\end{array}$ & 68 & 12 \\
\hline $\begin{array}{l}\text { Clinical } \\
\text { Elements }\end{array}$ & 67 & 13 \\
\hline $\begin{array}{l}\text { Clinical } \\
\text { Elements }\end{array}$ & 65 & 15 \\
\hline $\begin{array}{l}\text { Clinical } \\
\text { Elements }\end{array}$ & 65 & 15 \\
\hline $\begin{array}{l}\text { Clinical } \\
\text { Elements }\end{array}$ & 65 & 15 \\
\hline $\begin{array}{l}\text { Clinical } \\
\text { Elements }\end{array}$ & 65 & 15 \\
\hline $\begin{array}{l}\text { Clinical } \\
\text { Elements }\end{array}$ & 63 & 17 \\
\hline $\begin{array}{l}\text { Clinical } \\
\text { Elements }\end{array}$ & 62 & 18 \\
\hline $\begin{array}{l}\text { Clinical } \\
\text { Elements }\end{array}$ & 60 & 20 \\
\hline $\begin{array}{l}\text { Clinical } \\
\text { Elements }\end{array}$ & 60 & 20 \\
\hline
\end{tabular}

TABLE 3: Strongly prioritized topics: Diagnosis and Management Domain. 


\section{Cureus}

\begin{tabular}{|c|c|c|c|}
\hline Topic & Category theme & Score & $\begin{array}{l}\text { Overall rank } \\
\text { order }\end{array}$ \\
\hline $\begin{array}{l}\text { Physical performance during a code (such as chest compression or bag-valve mask } \\
\text { quality) }\end{array}$ & Procedural Skills & 79 & 1 \\
\hline Endotracheal intubation procedure & Procedural Skills & 75 & 5 \\
\hline Placement of hemodynamic monitoring devices (central venous and arterial lines) & Procedural Skills & 74 & 6 \\
\hline Mastery of a specific procedural skill & Procedural Skills & 73 & 7 \\
\hline Procedural training: individual steps & Procedural Skills & 73 & 7 \\
\hline Procedures: pericardiocentesis & Procedural Skills & 73 & 7 \\
\hline Ability to repeat skill/procedure more than once & Procedural Skills & 72 & 8 \\
\hline Establishing and maintaining a sterile field & Procedural Skills & 61 & 9 \\
\hline Familiarity with equipment & Procedural Skills & 70 & 10 \\
\hline Pleural drainage/chest tube placement & Procedural Skills & 70 & 10 \\
\hline Abdominal paracentesis & Procedural Skills & 69 & 11 \\
\hline General arrway management procedures except endotracheal Intubation & Procedural Skills & 69 & 11 \\
\hline Regional anesthesia procedures (nerve blocks or epidural placement) & Procedural Skills & 69 & 11 \\
\hline Swan Ganz placement & Procedural Skills & 67 & 13 \\
\hline Understanding the importance of each step of a procedure & $\begin{array}{l}\text { Conceptual } \\
\text { Elements }\end{array}$ & 67 & 13 \\
\hline Checklist usage for a procedure & $\begin{array}{l}\text { Conceptual } \\
\text { Elements }\end{array}$ & 66 & 14 \\
\hline Neurologic (e.g., burr hole) & Procedural Skills & 66 & 14 \\
\hline Use of point-of-care ultrasound & Procedural Skills & 66 & 14 \\
\hline Iroublesnooting auring a procedure & Procequral SKIIIs & 65 & 15 \\
\hline Procedures: performing a timeout & $\begin{array}{l}\text { Conceptual } \\
\text { Elements }\end{array}$ & 62 & 18 \\
\hline
\end{tabular}

TABLE 4: Strongly prioritized topics: Procedures Domain.

\begin{tabular}{|c|c|c|c|}
\hline Topic & Category theme & Score & $\begin{array}{l}\text { Overall } \\
\text { rank order }\end{array}$ \\
\hline Situational awareness during a code & $\begin{array}{l}\text { Conceptual } \\
\text { Elements }\end{array}$ & 79 & 1 \\
\hline Speaking up during resuscitation & $\begin{array}{l}\text { Interpersonal } \\
\text { Elements }\end{array}$ & 77 & 3 \\
\hline Team-specific general situational awareness & $\begin{array}{l}\text { Interpersonal } \\
\text { Elements }\end{array}$ & 77 & 3 \\
\hline Error recovery during team training & $\begin{array}{l}\text { Interpersonal } \\
\text { Elements }\end{array}$ & 76 & 4 \\
\hline Interprofessional team dynamics & $\begin{array}{l}\text { Interpersonal } \\
\text { Elements }\end{array}$ & 76 & 4 \\
\hline Leadership development during a code & $\begin{array}{l}\text { Interpersonal } \\
\text { Elements }\end{array}$ & 76 & 4 \\
\hline
\end{tabular}




\section{Cureus}

Team leader cognitive load during a code

Time management during mock codes

Algorithm application during a code (pediatric advanced life support, advanced cardiovascular life support)

Communicating bad news

Rapid decision-making during emergencies

Team training (e.g., team communication and coordination)

Interprofessional leadership

Understanding team roles and responsibilities including role identification and execution

Using standard communication tools (e.g., TeamSTEPPS, situation-backgroundassessment-recommendation/request)

Collaboration and sharing of concept knowledge during a code

Using debriefing as a teaching tool

Error detection during a code

Uncovering systems vulnerabilities during a code

Ability to maintain a "big picture" view

Avoidance of hierarchy issues

Avoiding cognitive bias when making diagnostic errors during a code

Contingency planning during a code

Debriefing after a code

Effective pre-briefing for codes

Patient assessment during a code

Avoidance of fixation

Communicating empathy to patients and families

Confidence building for trainees during a code

Competency managing and delegating procedures

Preparation during a code

\begin{tabular}{|c|c|}
\hline $\begin{array}{l}\text { Interpersonal } \\
\text { Elements }\end{array}$ & 76 \\
\hline $\begin{array}{l}\text { Interpersonal } \\
\text { Elements }\end{array}$ & 76 \\
\hline Clinical Elements & 75 \\
\hline $\begin{array}{l}\text { Interpersonal } \\
\text { Elements }\end{array}$ & 75 \\
\hline $\begin{array}{l}\text { Interpersonal } \\
\text { Elements }\end{array}$ & 75 \\
\hline $\begin{array}{l}\text { Interpersonal } \\
\text { Elements }\end{array}$ & 75 \\
\hline $\begin{array}{l}\text { Interpersonal } \\
\text { Elements }\end{array}$ & 74 \\
\hline $\begin{array}{l}\text { Interpersonal } \\
\text { Elements }\end{array}$ & 74 \\
\hline $\begin{array}{l}\text { Interpersonal } \\
\text { Elements }\end{array}$ & 74 \\
\hline $\begin{array}{l}\text { Interpersonal } \\
\text { Elements }\end{array}$ & 73 \\
\hline $\begin{array}{l}\text { Conceptual } \\
\text { Elements }\end{array}$ & 73 \\
\hline $\begin{array}{l}\text { Conceptual } \\
\text { Elements }\end{array}$ & 72 \\
\hline $\begin{array}{l}\text { Conceptual } \\
\text { Elements }\end{array}$ & 72 \\
\hline $\begin{array}{l}\text { Conceptual } \\
\text { Elements }\end{array}$ & 71 \\
\hline $\begin{array}{l}\text { Interpersonal } \\
\text { Elements }\end{array}$ & 71 \\
\hline $\begin{array}{l}\text { Conceptual } \\
\text { Elements }\end{array}$ & 71 \\
\hline $\begin{array}{l}\text { Conceptual } \\
\text { Elements }\end{array}$ & 71 \\
\hline $\begin{array}{l}\text { Interpersonal } \\
\text { Elements }\end{array}$ & 71 \\
\hline $\begin{array}{l}\text { Interpersonal } \\
\text { Elements }\end{array}$ & 71 \\
\hline Clinical Elements & 71 \\
\hline $\begin{array}{l}\text { Conceptual } \\
\text { Elements }\end{array}$ & 70 \\
\hline $\begin{array}{l}\text { Interpersonal } \\
\text { Elements }\end{array}$ & 70 \\
\hline $\begin{array}{l}\text { Conceptual } \\
\text { Elements }\end{array}$ & 69 \\
\hline $\begin{array}{l}\text { Interpersonal } \\
\text { Elements }\end{array}$ & 68 \\
\hline $\begin{array}{l}\text { Interpersonal } \\
\text { Elements }\end{array}$ & 68 \\
\hline
\end{tabular}




\section{Cureus}

Critical self-analysis during team training

$\begin{array}{lll}\begin{array}{l}\text { Conceptual } \\ \text { Elements }\end{array} & 67 & 13 \\ \begin{array}{l}\text { Interpersonal } \\ \text { Elements }\end{array} & 67 & 13 \\ \begin{array}{l}\text { Interpersonal } \\ \text { Elements }\end{array} & 66 & 14 \\ \begin{array}{l}\text { Interpersonal } \\ \text { Elements }\end{array} & 62 & 18 \\ \begin{array}{l}\text { Interpersonal } \\ \text { Elements }\end{array} & 61 & 19 \\ \text { Interpersonal } & 61 & 19 \\ \text { Elements } & & \end{array}$

Team training for palliative care/end-of-life coordination/medical futility Elements

TABLE 5: Strongly prioritized topics: Teamwork and Communication Domain.

Topics with moderate priority are listed in Table 6.

Topic

Deep knowledge of critical illness (beyond algorithms)

Overdoses and poisonings: medication indications and side effects (of drug(s) taken)

Anesthesia medication indications and effects (e.g., analgesic, sedatives, neuromuscular blockade)

Cardiopulmonary interactions

General analgesia, sedation, neuromuscular blockade

Declaration and management of brain death

Diagnosis and management of burns and drownings

Managing environmental emergencies (e.g., drowning, burns)

Approach to palliative liberation of an endotracheal tube

Neurologic diagnosis and management (e.g., cerebrovascular disease, seizures)

Approach to ethical issues (e.g., conflict of interest, patient privacy)

Diagnosis and management of altered mentation or coma (e.g., encephalopathy, coma, delirium)

Managing endocrine emergencies (e.g., diabetic ketoacidosis, thyroid storm, hypocalcemia)

Toxic ingestions (diagnosis and management)

Diagnosis and management of bites and envenomations

Managing compartment syndrome/pulseless extremity

Management of genetic/metabolic emergencies (e.g., metabolic crisis)

Surgical diagnoses and management(e.g., abdominal compartment syndrome)

Obtaining consent for a procedure

Echocardiography

\section{Score}

57

57

52

52

$51 \quad 28$

$57 \quad 23$

$57 \quad 23$

$57 \quad 23$

$55 \quad 24$

$55 \quad 24$

$54 \quad 25$

$54 \quad 25$

$54 \quad 25$

$54 \quad 25$

$53 \quad 26$

$53 \quad 26$

$52 \quad 27$

$52 \quad 27$

$59 \quad 21$

$58 \quad 22$

TABLE 6: Moderately prioritized topics.

Evaluation of the recommended topics yielded four major domains: Procedural Skills, Clinical Elements, Conceptual Elements, and Interpersonal Elements (Figure 2). 


\section{Discussion}

We developed simulation-based critical care education priorities through the consensus of simulation experts across all pediatric and adult critical care facets. In defining these domains and categories of core simulation-based critical care content, we hope to improve the prioritization of SBME within critical care. Identifying priority areas for simulation-based education may help stakeholders, including healthcare organizations, professional societies, and educational leadership, prioritize funding, budget allocations, faculty/staff support, research collaboratives, and program assessments. This content should aid training programs and simulation centers in evaluating learners and target learning needs specific to critical care medicine.

Prior work using the Delphi process for content generation has been used to generate UME content for critical care [15] and pediatric content for emergency medicine residents [14]. In both cases, the scope was different and not adequately specific to apply to critical care core competencies in the context of either graduate medical education or interprofessional education, again highlighting a need for this study. Not surprisingly, our results demonstrate strong agreement that simulation is well suited to teach several aspects of communication and procedural skills. A focus on aspects of cardiac arrest, shock, and other resuscitation scenarios was also noted to be important components of SBME in intensive care units. This study also demonstrates a potential role for SBME to teach more cognitive skills such as diagnosis and management of various clinical entities.

A key finding of our study was a generally good agreement on the topics for critical care education across teamwork, communication, and procedural skills. While pathophysiology and diagnosis may differ across the age spectrum and between different medical and surgical subspecialties, often the basics of procedures and especially the skills necessary for effective communication during resuscitation events transcend specialties and professions. This suggests that combining interprofessional critical care simulation programs across subspecialties may benefit from optimizing resource utilization. We hope that this will ultimately facilitate a shift from training in silos to training together through interprofessional simulations. In doing so, we might better approximate how we might create better learning activities for critical care teams.

Despite an underrepresentation of neurointensivist participation, neurological emergencies were identified as an area of high priority, potentially reflecting emerging awareness of the utility of SBME for teaching about neurological emergencies and technological advances that support creating such simulations. Intensivists, including pediatric, emergency medicine, and trauma critical care providers, have essential roles in the initial identification and management of acute neurological injury and thus may have ranked these topics higher.

One of the main challenges of the widespread adoption of simulation education is insufficient faculty time and the limited ability to regularly pull trainees and interprofessional colleagues from clinical care responsibilities. Especially during the global coronavirus disease 2019 (COVID-19) pandemic, any disruption to the available pool of faculty and trainees available to provide bedside care is unlikely to be supported and may result in the cancellation of simulation courses altogether. By co-training learners from different specialties, no individual department is left with inadequate patient care coverage. Additionally, if course goals and objectives are uniform, faculty across different specialties could share the responsibility of teaching sessions. Finally, expanding the pool of potential learners and research subjects can further facilitate the performance of simulation education research. Additionally, the COVID-19 pandemic has shown the importance of cross-training healthcare providers who are not typically critical care providers for such surges. This priority list helps healthcare systems and simulation centers determine key objectives for rapid just-in-time training.

Our work has several significant limitations, including a variable survey response rate and drop-off between rounds resulting in a small sample size of predominantly physicians and a higher ratio of pediatric-to-adult intensivists. Additionally, it is not possible to extrapolate if the opinions expressed by educators truly reflect learners' needs. Third, our results may have been different had this study been performed after the onset of the COVID-19 pandemic.

The Delphi method has inherent limitations within the process itself. The intention is to gather and obtain consensus based upon experts' opinions and insight. It does not result in an evidence-based summary (a Delphi is performed precisely because of a lack of evidence within a specific area). Additionally, the final product is a list of statements that gained consensus but does not provide additional detail or depth to those answers. This study's specific aims focused on overall expert opinion for which consensus was sought over a wide range of content, spanning multiple disciplines, several specialties, and age ranges, thus limiting the ability to obtain in-depth input for any specific subspecialty or pathology. However, the broad net that was cast in this project may also be seen as a strength as it increases the generalizability of the findings. Given the scope of the study, some of the topics listed by subspecialty boards were more detailed than others, leading to a wide range in the specificity of the topics included.

The Delphi survey's extensive and iterative nature could have led to potential survey fatigue and content bias. The composition of our expert panel may have been biased as well. Although we tried to mitigate this 
by broadly recruiting experts in the field through multiple lists, the final panel was somewhat self-selecting by answering our recruitment emails. Given that this Delphi process was conducted remotely, as opposed to in-person, bias from a smaller group of "influential" experts was less likely to have occurred. However, using a remote method without an in-person component may have also contributed to the relatively high attrition rate experienced. While we had a reasonably sized group, it is unclear from existing literature what the ideal size of an expert panel should be. A group should generally be large enough to propose new ideas and overcome attrition issues. A total of 30 members have been suggested as a reasonable target to attain these goals and align with our final survey round [23]. Finally, while we sought to have a broad representation of experts across disciplines within the United States, these results lack international representation and likely differ in other countries, where variations in educational curricula, competing interests, and different priorities may be present.

\section{Conclusions}

In conclusion, we defined priority areas in SBME curricula for critical care using a modified Delphi process. Healthcare systems and simulation centers should focus on these criteria when designing training curricula for critical care teams across medical professions and subspecialties and consider sharing resources between different groups of faculty and learners. As critical care practice changes with new medications, technology, and multispecialty teams, judicious use of simulation will help ensure timely and effective educational interventions that will also inform quality improvement and safety initiatives.

\section{Additional Information \\ Disclosures}

Human subjects: Consent was obtained or waived by all participants in this study. Animal subjects: All authors have confirmed that this study did not involve animal subjects or tissue. Conflicts of interest: In compliance with the ICMJE uniform disclosure form, all authors declare the following: Payment/services info: All authors have declared that no financial support was received from any organization for the submitted work. Financial relationships: All authors have declared that they have no financial relationships at present or within the previous three years with any organizations that might have an interest in the submitted work. Other relationships: All authors have declared that there are no other relationships or activities that could appear to have influenced the submitted work.

\section{Acknowledgements}

The authors would like to acknowledge the SCCM Research Section, Simulation and Education Committee for their support of this project.

\section{References}

1. Lopreiato JO, Sawyer T: Simulation-based medical education in pediatrics. Acad Pediatr. 2015, 15:134-42. 10.1016/j.acap.2014.10.010

2. Hippe DS, Umoren RA, McGee A, Bucher SL, Bresnahan BW: A targeted systematic review of cost analyses for implementation of simulation-based education in healthcare. SAGE Open Med. 2020, 8:2050312120913451. 10.1177/2050312120913451

3. Kester-Greene N, Hall AK, Walsh CM: [Simulation curricular content in postgraduate emergency medicine: a multicentre Delphi study]. CJEM. 2019, 21:667-75. 10.1017/cem.2019.348

4. Bessmann EL, Østergaard HT, Nielsen BU, et al.: Consensus on technical procedures for simulation-based training in anaesthesiology: a Delphi-based general needs assessment. Acta Anaesthesiol Scand. 2019, 63:720-9. 10.1111/aas.13344

5. Doughty CB, Kessler DO, Zuckerbraun NS, et al.: Simulation in pediatric emergency medicine fellowships. Pediatrics. 2015, 136:e152-8. 10.1542/peds.2014-4158

6. Manthous CA: On the outcome project. Yale J Biol Med. 2014, 87:213-20.

7. Seam N, Lee AJ, Vennero M, Emlet L: Simulation training in the ICU. Chest. 2019, 156:1223-33. 10.1016/j.chest.2019.07.011

8. Swing SR: The ACGME outcome project: retrospective and prospective . Med Teach. 2007, 29:648-54. 10.1080/01421590701392903

9. Kessler D, Pusic M, Chang TP, et al.: Impact of just-in-time and just-in-place simulation on intern success with infant lumbar puncture. Pediatrics. 2015, 135:e1237-46. 10.1542/peds.2014-1911

10. Barsuk JH, McGaghie WC, Cohen ER, O'Leary KJ, Wayne DB: Simulation-based mastery learning reduces complications during central venous catheter insertion in a medical intensive care unit. Crit Care Med. 2009, 37:2697-701.

11. Barsuk JH, McGaghie WC, Cohen ER, Balachandran JS, Wayne DB: Use of simulation-based mastery learning to improve the quality of central venous catheter placement in a medical intensive care unit. J Hosp Med. 2009, 4:397-403. 10.1002/jhm.468

12. Cohen ER, Feinglass J, Barsuk JH, Barnard C, O'Donnell A, McGaghie WC, Wayne DB: Cost savings from reduced catheter-related bloodstream infection after simulation-based education for residents in a medical intensive care unit. Simul Healthc. 2010, 5:98-102. 10.1097/SIH.0b013e3181bc8304

13. Zackoff MW, Real FJ, Sahay RD, et al.: Impact of an immersive virtual reality curriculum on medical students' clinical assessment of infants with respiratory distress. Pediatr Crit Care Med. 2020, 21:477-85. 10.1097/PCC.0000000000002249 


\section{Cureus}

14. Mitzman J, King AM, Fastle RK, et al.: A modified Delphi study for development of a pediatric curriculum for emergency medicine residents. AEM Educ Train. 2017, 1:140-50. 10.1002/aet2.10021

15. Smith AG, Brainard JC, Campbell KA: Development of an undergraduate medical education critical care content outline utilizing the Delphi method. Crit Care Med. 2020, 48:98-103.

10.1097/CCM.0000000000004086

16. Humphrey-Murto S, Wood TJ, Gonsalves C, Mascioli K, Varpio L: The Delphi method. Acad Med. 2020, 95:168. 10.1097/ACM.0000000000002887

17. Johnston L, Sawyer T, Nishisaki A, et al.: Neonatal intubation competency assessment tool: development and validation. Acad Pediatr. 2019, 19:157-64. 10.1016/j.acap.2018.07.008

18. Brown BB: Delphi process: a methodology used for the elicitation of opinions of experts . RAND Corporation, Santa Monica, CA; 1968.

19. Lioce L, Lopreiato J, Downing D, et al.: Healthcare simulation dictionary. Agency for Healthcare Research and Quality, Rockville, MD; 2020. 10.23970/simulationv2

20. Johnson TP: Snowball sampling: introduction. John Wiley \& Sons, Hoboken, NJ; 2014 10.1002/9781118445112.stat05720

21. Lynn MR: Determination and quantification of content validity. Nurs Res. 1986, 35:382-5.

22. Altschuld JW, Thomas PM: Considerations in the application of a modified scree test for Delphi survey data . Eval Rev. 2016, 15:179-88. 10.1177/0193841x9101500201

23. Murphy MK, Black NA, Lamping DL, McKee CM, Sanderson CF, Askham J, Marteau T: Consensus development methods, and their use in clinical guideline development. Health Technol Assess. 1998, 2:i-iv, $1-88$. 